\title{
Thulium-doped Sodium Zinc Molybdenum Tellurite Glasses
}

\author{
Jin Zheng ${ }^{a}$ and ShiQing Man*b
}

School of Physics and Electronic Information, Yunnan Normal University, Kunming, Yunnan, China

ajinzheng620713@sina.com, b man_shiqing@yahoo.com

Keywords: Judd-Ofelt Theory; Rare-earth lon; Optical Transitions

\begin{abstract}
Researchers propose and demonstrate Sodium Zinc Molybdenum Tellurite glasses (Na2O-ZnO-MoO3-TeO2 or NZMT) as suitable hosts for rare earth ion thulium (Tm3+) operating at $1.47 \mu \mathrm{m}$ wavelength. The density, the refractive indices, the optical absorption have been measured. The Judd-Ofelt intensity parameters $\Omega 2, \Omega 4, \Omega 6$, the radiative rates, the branching ratios, and the fluorescence lifetimes were calculated according to Judd-Ofelt theory. Intense $1.47 \mu \mathrm{m}$ fluorescence was observed in these glass systems under 973-nm excitation. The bandwidth of the $3 \mathrm{H} 4 \rightarrow 3 \mathrm{~F} 4$ transition in NZMT glass is $\sim 100 \mathrm{~nm}$, which is significantly larger than that of fluoride glasses but less than that in KBG glasses. Measured fluorescence lifetimes of the $3 \mathrm{~F} 4 \rightarrow 3 \mathrm{H} 6$ and $3 \mathrm{H} 4 \rightarrow 3 \mathrm{~F} 4$ decay at room temperature were 2.842 and $0.323 \mathrm{~ms}$ respectively, and their quantum efficiencies were approximately $100 \%$ and $88.9 \%$. From the data, it appears that Tm3+ doped NZMT glasses are promising materials for waveguide lasers and amplifiers operation at 1.40-1.52 $\mu \mathrm{m}$ wavelength range.
\end{abstract}

\section{Introduction}

Tellurite glasses combine with the attributes of wide transmission region $(0.35-6 \mu \mathrm{m})$, good glass stability, rare-earth ion solubility, slow corrosion rate, lowest phonon energy spectrum among oxide glass formers, high refractive index, and high nonlinear refractive index [1]. Neodymium $(\mathrm{Nd} 3+)$-doped tellurite singlemode fiber laser and Er3+-doped tellurite singlemode fiber laser have been demonstrated recently[2,3]. The signal-gain characteristics of Er3+ doped tellurite fiber amplifiers are clarified based on spectroscopic properties and signal-gain measurements [4].

The complete low-loss window of silica-based optical fibers ranges from 1450 to $1650 \mathrm{~nm}$. However, only part of the wavelength regions in the $1550 \mathrm{~nm}$ range have been used in wavelength division multiplexing (WDM) networks, which is limited by the operating wavelength and bandwidth of erbium doped fiber amplifier (EDFA). To support the need for higher capacity and increased flexibility bandwidth expansion in WDM networks is attractive. Thus, rare earth doped planar waveguides and fiber devices operating at the $1.4 \mu \mathrm{m}$ wavelength range will be very useful. Tm3+ ion has been shown to provide gain in the 1450-1510 nm window; this is very desirable because it is the next lowest loss window to the erbium $(\mathrm{Er} 3+)$ emission. Tm3+ ion for $1.47 \mu \mathrm{m}$ emission has been investigated in a variety of glasses [5-8]. A maximum signal gain of $18 \mathrm{~dB}$ has been achieved in a $20 \mathrm{~m}$ long Tm3+ doped fiber at a signal wavelength of $1.46 \mu \mathrm{m}$ for a pump power of $150 \mathrm{~mW}$ at $0.79 \mu \mathrm{m}$ [9].

In this paper, Tm3+ doped NZMT glasses suitable for $1.47 \mu \mathrm{m}$ wavelength operation have been demonstrated. Absorption, emission, radiative lifetimes, and non-radiative properties of the glasses were investigated. 


\section{Experiments}

Tm3+-doped glasses were prepared from anhydrous sodium carbonate (Na2CO3), zinc oxide $(\mathrm{ZnO})$, molybdenum oxide (MoO3), and tellurium oxide ( $\mathrm{TeO} 2)$ powders. All this powders (99.999-99.5\% purity) were obtained from Strem Chemicals Company. The glasses samples used in this research have the following molar composition: 10Na2O-10ZnO-10MoO3-70TeO2. Tm3+-doped NZMT glasses were prepared by doping $1.0 \mathrm{wt} \% \mathrm{Tm} 2 \mathrm{O} 3$ into the NZMT glass raw chemicals. The glass powders are melted in a platinum crucible by an electrically heated furnace at $750^{\circ} \mathrm{C}$. The glasses were subsequently annealed at lower temperatures, and then sliced and polished to dimensions of $20 \mathrm{~mm} \times 20 \mathrm{~mm} \times 2 \mathrm{~mm}$.

The density of this sample is $5.31 \mathrm{~g} / \mathrm{cm} 3$. By using the Metricon 2010 prism coupler technique at three wavelengths the refractive indices were obtained. The NZMT glasses refractive indices were 1.950 and 2.011 at $633 \mathrm{~nm}$ and $1550 \mathrm{~nm}$ wavelength respectively.

Using Cary 5000 double-bean spectrophotometer the absorption spectra were obtained from $300 \mathrm{~nm}$ to $2600 \mathrm{~nm}$. The fluorescence spectra were measured with a SPEX 500M monochromator and detected by a liquid-nitrogen-cooled germanium detector. The fluorescence lifetime of the 3F4 and $3 \mathrm{H} 4$ level of $\mathrm{Tm} 3+$ ion is measured with a $980 \mathrm{~nm}$ laser diode light pulses and an InGaAs photodetector.

\section{Results and Discussion}

The absorption spectrum of Tm3+ doped tellurite glasses is shown in Fig. 1, from which an energy-level diagram of $\mathrm{Tm} 3+$ is constructed (Fig. 2). The absorption bands can be ascribed to transitions from the ground state (3H6) to the excited states of the Tm3+ ion. The band assignments are also indicated in this figure. The absorption spectra show wavelengths that may be used as pump lights for laser and optical amplifiers. Four possible pump transitions are $3 \mathrm{H} 6 \rightarrow 1 \mathrm{G} 4$ (465 nm), $3 \mathrm{H} 6 \rightarrow 3 \mathrm{~F} 2(658 \mathrm{~nm}), 3 \mathrm{H} 6 \rightarrow 3 \mathrm{~F} 3(687 \mathrm{~nm})$ and $3 \mathrm{H} 6 \rightarrow 3 \mathrm{~F} 4(793 \mathrm{~nm})$.

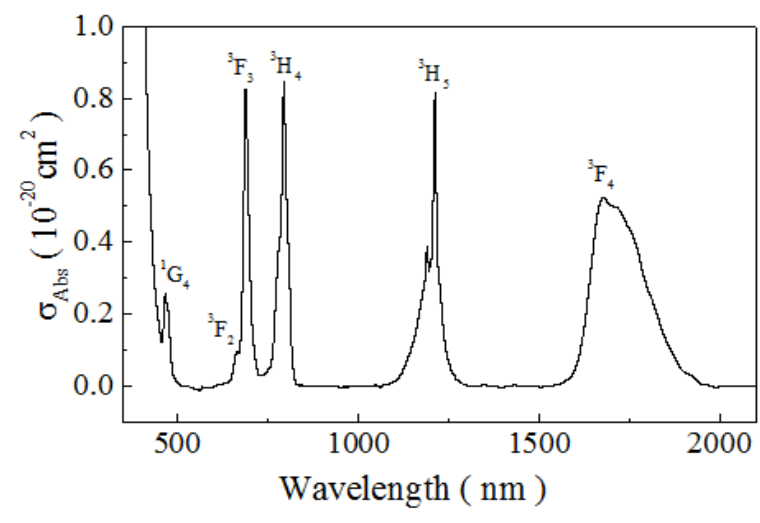

Fig. 1 Absorption cross section of $\mathrm{Tm}^{3+}$ in NZMT glasses

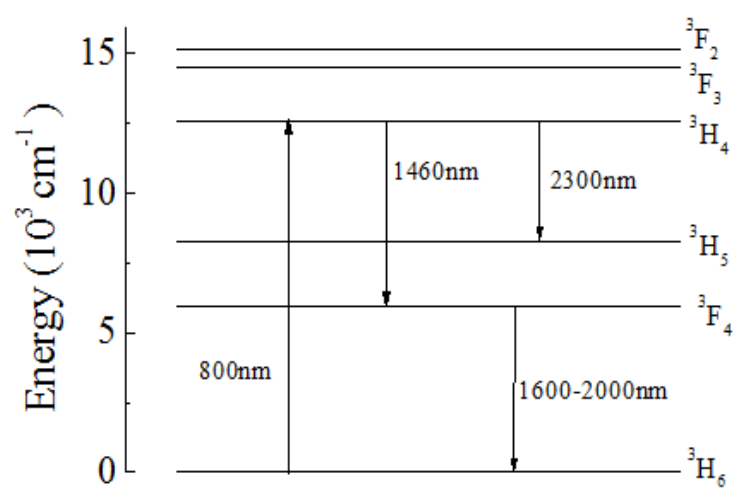

Fig. 2 Energy level diagram of $\mathrm{Tm}^{3+}$ in NZMT glass

A Judd-Ofelt analysis is employed to determine the radiative transition within the $4 \mathrm{f} n$ configuration of a rare earth ion [10,11]. The emission probability of electric dipole transition is dominated by the Judd-Ofelt $\Omega \mathrm{t}$ parameters [12], which are functions of crystal field and radial integral of $4 \mathrm{f}$ and $5 \mathrm{~d}$ electrons. The line strength for an electric-dipole transition between a ground state $\mathbf{J}$ and an excited state $\mathbf{J}$ ' is calculated as 


$$
S_{e d}\left[J ; J^{\prime}\right]=\sum_{t=2,4,6} \Omega_{t}\left\langle\alpha S L, J\left\|U^{(t)}\right\| \alpha^{\prime} S^{\prime} L^{\prime}, J^{\prime}\right\rangle^{2},
$$

(1)

where $\Omega_{\mathrm{t}}$ is the Judd-Ofelt parameters, $J$ is the total angular momentum of initial state, $J^{\prime}$ is the total angular momentum of final states, $\alpha \mathrm{SL}$ is defined all other quantum numbers needed to specify the states and $<\left\|U^{(t)}\right\|>$ is denoted the double reduced matrix elements of unit tensor operators for the corresponding transition.

From the absorption spectrum, the measured oscillator strength fmeas of the transitions are obtained by integrating absorption coefficients for each band using the following expression

$$
f_{\text {meas }}=\frac{2303 m c}{\pi e^{2} N_{A}} \int(v) d v=4.318 \times 10^{-9} \int(v) d v
$$

where $\mathrm{m}$ is the mass of the electron, $\mathrm{e}$ is the charge of the electron, $\mathrm{c}$ is the velocity of light, $\varepsilon(v)$ is the molar absorption coefficient, $v$ is the light frequency and $\mathrm{N}_{\mathrm{A}}$ is the Avogadro number. The experimental oscillator strengths of the transitions from the ground ${ }^{3} \mathrm{H}_{6}$ level to seven excited levels are determined by numerical integration of the corresponding absorption bands in Fig. 1.

The refractive indices of the glasses are measured using prism coupler 2010 with a prism $(2.0<\mathrm{n}>2.4)$. The refractive indices at $633 \mathrm{~nm}$ and $1550 \mathrm{~nm}$ are 2.0112 and 1.9261 , respectively. Since the experimental oscillator strengths contain electric-dipole and magnetic-dipole contributions, the magnetic-dipole contribution is subtracted from the experimental oscillator strengths to obtain the magnetic-dipole contribution. The magnetic-dipole contribution, fmd, is obtained from the refractive index of the investigated glasses and the quantities, f [13]. For each wavelength, the refractive index was calculated using Cauchy's relation: using Cauchy's relation:

(3)

$$
n=A+\frac{B}{\lambda^{2}}
$$

$$
\begin{aligned}
\mathrm{A}=1.9092, \mathrm{~B} & =40870 \times 104 \mathrm{~nm} 2 \\
f_{m d} & =n f^{\prime},
\end{aligned}
$$

The relation between line strength $S_{\text {ed }}$ and oscillator strength $f$ for each electric dipole transition (at average frequency $v$ ) is given by

(5)

$$
f_{\text {calc }}\left(J, J^{\prime}\right)=\frac{8 \pi^{2} m c\left(n^{2}+2\right)^{2}}{3 h \bar{\lambda}(2 J+1) 9 n} S_{e d}\left(J ; J^{\prime}\right),
$$

Where $\mathrm{h}$ is Planck's constant, $\mathrm{n}$ is the refractive index of the host, $\bar{\lambda}$ is the mean wavelength of the transition. From the integrated absorbance, three intensity parameters $\Omega_{t}(t=2,4,6)$ were determined by using a least-square fitting approach.

Accuracy of the least-squares approximation was evaluated by the root mean-square (rms) deviation from the measured oscillator strengths, Which is defined as

$$
r m s=\left(\frac{\sum\left(f_{\text {meas }}-f_{\text {cal }}\right)^{2}}{\text { no. of transition-no. of parameters }}\right)^{1 / 2} .
$$


The measured and calculated oscillator strengths, the electric-dipole line strengths $S_{\text {ed }}$ for some transitions, and the Judd-Ofelt intensity parameters of of $\mathrm{Tm}^{3+}$-doped NZMT glasses are presented in TABLE I. The Judd-Ofelt intensity parameters $\Omega_{\mathrm{t}}$, determined by using a least-squares fitting approach, are found to be $\Omega_{2}=4.74 \times 10^{-20} \mathrm{~cm}^{2}, \Omega_{4}=1.20 \times 10^{-20} \mathrm{~cm}^{2}$, and $\Omega_{6}=1.33 \times 10^{-20} \mathrm{~cm}^{2}$.

Table 1 The measured and calculated oscillator strengths, the electric-dipole line strengths Sed for some transitions, and the Judd-Ofelt intensity parameters of $\mathrm{Tm}^{3+}$ in NZMT glasses

\begin{tabular}{cccccc}
\hline Transition & Energy $\left[\mathbf{c m}^{-\mathbf{1}}\right]$ & $\boldsymbol{f}_{\text {exp }}\left[\mathbf{1 0}^{-\mathbf{6}}\right]$ & $\boldsymbol{f}_{\text {cal }}\left[\mathbf{1 0}^{-\mathbf{6}}\right]$ & $\boldsymbol{f}_{\boldsymbol{m} \boldsymbol{d}}\left[\mathbf{1 0}^{-\mathbf{6}}\right]$ & $\boldsymbol{S}_{\boldsymbol{e d}}\left[\mathbf{1 0}^{\mathbf{- 2 0}}\right]$ \\
\hline${ }^{3} \mathrm{H}_{6} \rightarrow{ }^{3} \mathrm{~F}_{4}$ & 5961 & 3.4913 & 3.4835 & & 3.7394 \\
${ }^{3} \mathrm{H}_{6} \rightarrow{ }^{3} \mathrm{H}_{5}$ & 8254 & 2.5048 & 2.1356 & 0.5278 & 1.5125 \\
${ }^{3} \mathrm{H}_{6} \rightarrow{ }^{3} \mathrm{H}_{4}$ & 12602 & 4.2463 & 4.1879 & & 2.0631 \\
${ }^{3} \mathrm{H}_{6} \rightarrow{ }^{3} \mathrm{~F}_{3,2}$ & 14513 & 4.7364 & 4.6023 & & 1.8726 \\
${ }^{3} \mathrm{H}_{6} \rightarrow{ }^{1} \mathrm{G}_{4}$ & 21482 & 1.3537 & 1.3261 & & 0.3480 \\
$\Omega_{2}\left[10^{-20} \mathrm{~cm}^{2}\right]$ & & & & 4.74 & \\
$\Omega_{4}\left[10^{-20} \mathrm{~cm}^{2}\right]$ & & & 1.20 & \\
$\Omega_{6}\left[10^{-20} \mathrm{~cm}^{2}\right]$ & & & 1.31 & \\
Root-mean-square deviation $\left[10^{-6}\right]$ & & & 0.154 & \\
\hline
\end{tabular}

By using the Judd-Ofelt intensity parameter $\Omega_{\mathrm{t}}$, some important radiative properties are calculated. The radiative transitions probabilities A from the $J$ to the $J$ ' levelsis calculated from the following equation [14]

$$
A\left[(S, L) J:\left(S^{\prime}, L^{\prime}\right) J^{\prime}\right]=\frac{64 \pi^{4} e^{2} n}{3 h(2 J+1) \bar{\lambda}^{3}}\left[\frac{\left(n^{2}+2\right)^{2}}{9}\right] \sum_{t=2,4,6} \Omega_{t}\left\langle(S, L) J\left\|U^{(t)}\right\|\left(S^{\prime}, L^{\prime}\right) J^{\prime}\right\rangle^{2},
$$

where $\mathrm{h}$ is Planck Constant, e is the charge of the electro $\mathrm{n}, \mathrm{n}$ is the refractive index, $\lambda$ is the mean wavelength of the transition, $J$ is the total angular momentum of the initial state, $\langle\|U\|\rangle$ is the double reduced matrix element of unit tensor operators calculated in the intermediate-coupling approximation.

The branching ratios for the different transitions from $\mid(\mathrm{S}, \mathrm{L}) J$ to lower level $\left(\mathrm{S}, \mathrm{L}^{\prime}\right) J^{\prime} \mid$ is given by

$$
\beta\left[(S, L) J:\left(S^{\prime}, L^{\prime}\right) J^{\prime}\right]=\frac{A\left[(S, L) J:\left(S^{\prime}, L^{\prime}\right) J^{\prime}\right]}{\sum_{S L^{\prime}, J^{\prime}} A\left[(S L J, S) L^{\prime}, \delta\right]},
$$

The radiative lifetime is expressed as the following equation

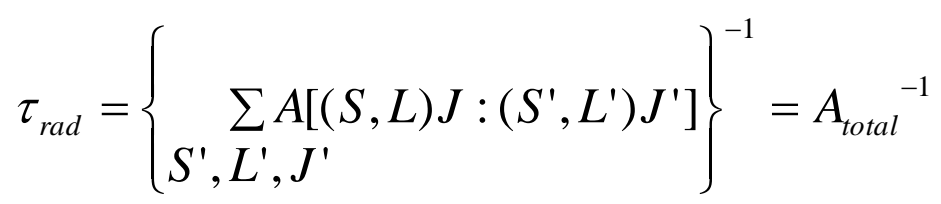

The quantum efficiency of the $\mid(S, L) J>$ manifold is defined as

$$
\eta=\tau_{\text {meas }} / \tau_{\text {rad }}
$$

where $\tau_{\text {meas }}$ and $\tau_{\text {rad }}$ are the experimentally determined and radiative lifetime, respectively.

Table II gives the spontaneous transition probabilities $A$, the branching ratios $\mathrm{b}$, and the calculated lifetimes $\tau_{\text {rad }}$ in $\mathrm{Tm}^{3+}$-doped NZMT glasses。 
Fig. 3 shows the emission spectra of $\mathrm{Tm}^{3+}$ doped NZMT glass in the range of 1300-1650 nm. Fluorescence bands at $1.47 \mu \mathrm{m}\left({ }^{3} \mathrm{H}_{4} \rightarrow{ }^{3} \mathrm{~F}_{4}\right)$ was observed from the glass doped with 1.0 wt. \% $\mathrm{Tm}_{2} \mathrm{O}_{3}$. Because of limitations of the spectrometer, only part of the spectrum of the the ${ }^{3} \mathrm{~F}_{4} \rightarrow{ }^{3} \mathrm{H}_{6}$ transition in the 1600-2000 nm can be observed. The bandwidth of the ${ }^{3} \mathrm{H}_{4} \rightarrow{ }^{3} \mathrm{~F}_{4}$ transition is $\sim 100$ $\mathrm{nm}$, which is significantly larger than that of fluoride glasses but less than that in KBG glasses $[15,16]$

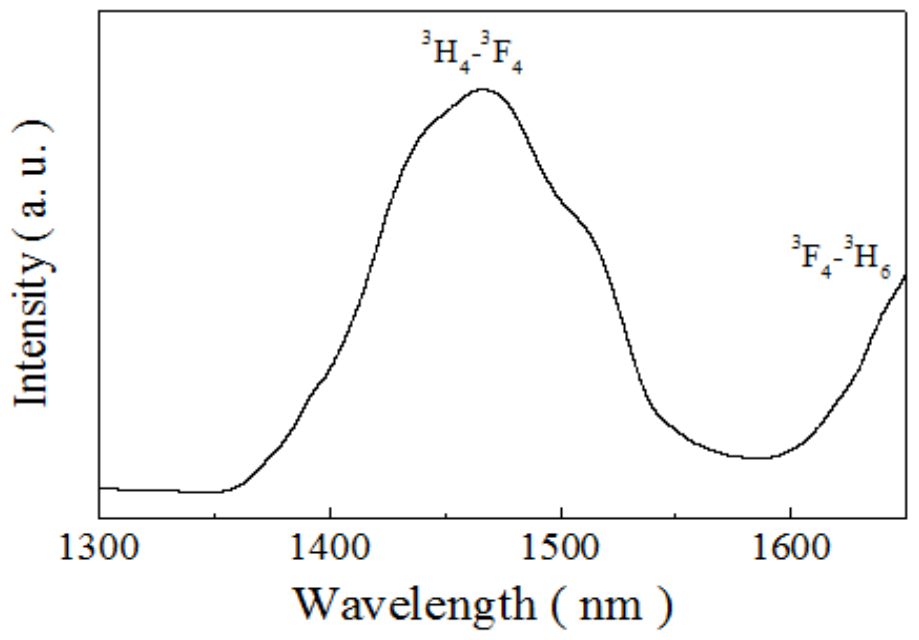

Fig. 3 Fluorescence spectrum of $\mathrm{Tm}^{3+}$ doped NZMT

glass in the range of $1300-1650 \mathrm{~nm}$

Measured fluorescence lifetimes of the ${ }^{3} \mathrm{~F}_{4} \rightarrow{ }^{3} \mathrm{H}_{6}$ and ${ }^{3} \mathrm{H}_{4} \rightarrow{ }^{3} \mathrm{~F}_{4}$ decay at room temperature were 2.842 and $0.323 \mathrm{~ms}$ respectively, and their quantum efficiencies were approximately $100 \%$ and $88.9 \%$. From the data, it appears that $\mathrm{Tm}^{3+}$-doped NZMT glasses are promising materials for use in lasers and optical amplifiers operating in the $1.47 \mu \mathrm{m}$ wavelength range.

Table 2 Calculated Spontaneous Emission Probabilities, Branching Ratios, and Lifetimes of $\mathrm{Tm}^{3+}$-doped NZMT

\begin{tabular}{ccccccc}
\hline Transition & $\bar{\lambda}[\mathbf{n m}]$ & $\boldsymbol{A}_{\text {ed }}\left[\mathbf{s}^{-\mathbf{1}}\right]$ & $\boldsymbol{A}_{\boldsymbol{m} \boldsymbol{d}}\left[\mathbf{s}^{\mathbf{- 1}}\right]$ & $\boldsymbol{A}_{\text {total }}\left[\mathbf{s}^{\mathbf{- 1}}\right]$ & $\boldsymbol{\beta}_{\boldsymbol{i j}}$ & $\boldsymbol{\tau}_{\text {rad }}[\boldsymbol{\mu} \mathbf{s}]$ \\
\hline${ }^{1} \mathrm{D}_{2} \rightarrow{ }^{1} \mathrm{G}_{4}$ & 1486 & 323 & & 323 & 0.004 & 13 \\
${ }^{1} \mathrm{D}_{2} \rightarrow{ }^{3} \mathrm{~F}_{2}$ & 778 & 1590 & 61 & 1651 & 0.022 & \\
${ }^{1} \mathrm{D}_{2} \rightarrow{ }^{3} \mathrm{~F}_{3}$ & 745 & 2348 & 101 & 2449 & 0.032 & \\
${ }^{1} \mathrm{D}_{2} \rightarrow{ }^{3} \mathrm{H}_{4}$ & 657 & 3768 & & 3768 & 0.049 & \\
${ }^{1} \mathrm{D}_{2} \rightarrow{ }^{3} \mathrm{H}_{5}$ & 504 & 241 & & 241 & 0.003 & \\
${ }^{1} \mathrm{D}_{2} \rightarrow{ }^{3} \mathrm{~F}_{4}$ & 450 & 48673 & & 48673 & 0.638 & \\
${ }^{1} \mathrm{D}_{2} \rightarrow{ }^{3} \mathrm{H}_{6}$ & 357 & 19187 & & 19187 & 0.251 & \\
${ }^{1} \mathrm{G}_{4} \rightarrow{ }^{3} \mathrm{~F}_{2}$ & 1634 & 16 & & 16 & 0.004 & 219 \\
${ }^{1} \mathrm{G}_{4} \rightarrow{ }^{3} \mathrm{~F}_{3}$ & 1494 & 67 & 4 & 71 & 0.016 & \\
${ }^{1} \mathrm{G}_{4} \rightarrow{ }^{3} \mathrm{H}_{4}$ & 1177 & 405 & 34 & 439 & 0.096 & \\
${ }^{1} \mathrm{G}_{4} \rightarrow{ }^{3} \mathrm{H}_{5}$ & 778 & 1276 & 154 & 1430 & 0.313 & \\
${ }^{1} \mathrm{G}_{4} \rightarrow{ }^{3} \mathrm{~F}_{4}$ & 646 & 294 & 9 & 303 & 0.066 & \\
${ }^{1} \mathrm{G}_{4} \rightarrow{ }^{3} \mathrm{H}_{6}$ & 470 & 2314 & & 2314 & 0.506 & \\
${ }^{3} \mathrm{~F}_{2} \rightarrow{ }^{3} \mathrm{~F}_{3}$ & 17513 & 0 & 0 & 0 & 0 & 393
\end{tabular}




\begin{tabular}{|c|c|c|c|c|c|c|}
\hline${ }^{3} \mathrm{~F}_{2} \rightarrow{ }^{3} \mathrm{H}_{4}$ & 4215 & 13 & & 13 & 0.005 & \\
\hline${ }^{3} \mathrm{~F}_{2} \rightarrow{ }^{3} \mathrm{H}_{5}$ & 1506 & 265 & & 266 & 0.105 & \\
\hline${ }^{3} \mathrm{~F}_{2} \rightarrow{ }^{3} \mathrm{~F}_{4}$ & 1069 & 1038 & & 1038 & 0.408 & \\
\hline${ }^{3} \mathrm{~F}_{2} \rightarrow{ }^{3} \mathrm{H}_{6}$ & 670 & 1225 & & 1225 & 0.482 & \\
\hline${ }^{3} \mathrm{~F}_{3} \rightarrow{ }^{3} \mathrm{H}_{4}$ & 5552 & 5 & & 5 & 0.001 & 216 \\
\hline${ }^{3} \mathrm{~F}_{3} \rightarrow{ }^{3} \mathrm{H}_{5}$ & 1558 & 647 & & 647 & 0.139 & \\
\hline${ }^{3} \mathrm{~F}_{3} \rightarrow{ }^{3} \mathrm{~F}_{4}$ & 1138 & 117 & 63 & 180 & 0.039 & \\
\hline${ }^{3} \mathrm{~F}_{3} \rightarrow{ }^{3} \mathrm{H}_{6}$ & 686 & 3806 & & 3806 & 0.820 & \\
\hline${ }^{3} \mathrm{H}_{4} \rightarrow{ }^{3} \mathrm{H}_{5}$ & 2166 & 27 & 11 & 33 & 0.014 & 363 \\
\hline${ }^{3} \mathrm{H}_{4} \rightarrow{ }^{3} \mathrm{~F}_{4}$ & 1432 & 187 & 24 & 211 & 0.077 & \\
\hline${ }^{3} \mathrm{H}_{4} \rightarrow{ }^{3} \mathrm{H}_{6}$ & 784 & 2504 & & 2504 & 0.910 & \\
\hline${ }^{3} \mathrm{H}_{5} \rightarrow{ }^{3} \mathrm{~F}_{4}$ & 4226 & 12 & 1 & 13 & 0.025 & 1836 \\
\hline${ }^{3} \mathrm{H}_{5} \rightarrow{ }^{3} \mathrm{H}_{6}$ & 1225 & 447 & 84 & 531 & 0.975 & \\
\hline${ }^{3} \mathrm{~F}_{4} \rightarrow{ }^{3} \mathrm{H}_{6}$ & 1725 & 361 & & 361 & 1 & 2770 \\
\hline
\end{tabular}

\section{Conclusions}

The optical properties of $\mathrm{Tm}^{3+}$ doped NZMT glassglasses are investigated. The $\mathrm{Tm}^{3+} 1.47 \mu \mathrm{m}$ emission is very desirable since it is the next lowest loss to the $\mathrm{Er}^{3+}$ window. The bandwidth of the ${ }^{3} \mathrm{H}_{4} \rightarrow{ }^{3} \mathrm{~F}_{4}$ transition in tellurite glass is $\sim 100 \mathrm{~nm}$, which is significantly larger than that of fluoride glasses but less than that in KBG glasses. Measured fluorescence lifetimes of the ${ }^{3} \mathrm{~F}_{4} \rightarrow{ }^{3} \mathrm{H}_{6}$ and ${ }^{3} \mathrm{H}_{4} \rightarrow{ }^{3} \mathrm{~F}_{4}$ decay at room temperature were 2.842 and $0.323 \mathrm{~ms}$ respectively, and their quantum efficiencies were approximately $100 \%$ and $88.9 \%$. Those results indicate that $\mathrm{Tm}^{3+}$ doped KBG glass is a promising candidate for optical amplifier operation at $1.40-1.52 \mu \mathrm{m}$.

\section{Acknowledgment}

This work is supported by the National Natural Science Foundation of China (NSFC) (Grant No. 21171072 and Grant No. 21361028).

\section{References}

[1] J.S. Wang, E.M. Vogel and E. Snitzer, "Tellurite glass: a new candidate for fiber devices", Opt. Mat. 3, 187 - 203 (1994).

[2] J.S. Wang, D.P. Machewirch, F. Wu, E.M. Vogel, and E. Snitzer, "Spectroscopic properties of neodymium-doped tellurite glass fiber ", Opt. Lett., 19, 1448-1449(1994)..

[3] A. Mori, Y. Ohishi and S. Sudo, "Er3+ doped ultra-transparent oxy-fluoride glass-ceramics for application in the $1.54 \mu \mathrm{m}$ telecommunication window",Electron. Lett., 33, 863. (1997),

[4] Y. Ohishi, A. Mori, M. Yamada, H. Ono, Y. Nishida, and K. Oikawa, "Nonlinear optical properties of Er2O3-doped 75Nb2O5-20TeO2-5ZnO glasses”, Opt. Lett., 23- (1998), 274.

[5] J.Y. Allain, M. Monerie, and H. Poignant, "Tunable CW lasing around 0.82, 1.48, 1.88 and 2.35 $\mu \mathrm{m}$ in thulium-doped fluorozirconate fibre”, Electron. Lett. 25, 1660-1662 (1989). 
[6] J.S. Wang, E. Snitzer, E.M. Vogel, and G.H. Sigel, Jr., "1.47, 1.88 and $2.8 \mu \mathrm{m}$ emissions of Tm3+ and Tm3+Ho3+-codoped tellurite glasses”, J. Lumin. 60\&61, 145-149 (1994).

[7] Y.B. Shin, W.Y. Cho, and J. Heo, "Multiphonon and cross relaxation phenomena in Ge-As (or Ga)-S glasses doped with Tm3+”, J. Non-Cryst. Solids, 208, 29-35 (1996).

[8] J. Heo, Y.B. Shin, and J.N. Jang, "Spectroscopic analysis of Tm3+ in PbO-Bi2O3-Ga2O3 glass", Appl. Opt. 34, 4284-4289 (1995).

[9] T. Sakamoto, M. Shimizu, T. Kanamori, Y. Terunuma, Y. Ohishi, M. Yamada, and S. Sudo, "1.4- $\mu \mathrm{m}$-band gain characteristics of a Tm-Ho-doped ZBLYAN fiber amplifier pumped in the 0.8- $\mu$ m band", IEEE Photon. Technol. Lett. 7, 983-985 (1995).

[10] B.R. Judd, "Optical absorption intensities of rare-earth ions”, Phys. Rev. 127, 750-761 (1962).

[11] G.S. Ofelt, "Intensities of crystal spectra of rare-earth ions", J. Chem. Phys. 37, 511-520 (1962).

[12] B.G. Wybourne, "Spectroscopic Properties of Rare Earth", Interscience, New York ,1965.

[13] W.T. Carnall, P. R. Fields, K. Rajnak, "Radiative and non-radiative transitions of rare-earth ions in glasses", J. Chem. Phys. 49, p.4412

[14] J. Sanz, R. Cases, R. Alcala, "Optical properties of Tm3+ in fluorozirconate glass", J. Non-Cryst. Solids, 93, 377-386 (1987)

[15] J. L. Adam, “Lanthanides in nonoxide glasses,” Chem. Rev. 102, 2461-2476(2002).

[16] S.Q. Man, E.Y.B. Pun, and P.S. Chung, "1.47- $\mu \mathrm{m}$ emission and multiphonon relaxation of $\mathrm{Tm} 3+$ ions in potassium bismuth gallate glasses" Journal of the Optical Society of America B, 21, 313-317 (2004). 